\title{
On the size of coset unions
}

\author{
Benjamin Sambale ${ }^{1}$ (D) Marius Tărnăuceanu ${ }^{2}$
}

Received: 13 December 2020 / Accepted: 6 October 2021 / Published online: 23 October 2021

(c) The Author(s) 2021

\begin{abstract}
Let $g_{1} H_{1}, \ldots, g_{n} H_{n}$ be cosets of subgroups $H_{1}, \ldots, H_{n}$ of a finite group $G$ such that $g_{1} H_{1} \cup \ldots \cup g_{n} H_{n} \neq G$. We prove that $\left|g_{1} H_{1} \cup \ldots \cup g_{n} H_{n}\right| \leq \gamma_{n}|G|$ where $\gamma_{n}<1$ is a constant depending only on $n$. In special cases, we show that $\gamma_{n}=\left(2^{n}-1\right) / 2^{n}$ is the best possible constant with this property and we conjecture that this is generally true.
\end{abstract}

Keywords Union of cosets · Subgroup covering · Conjecture

Mathematics Subject Classification 20D30 - 20D60 - 05E16

\section{Introduction}

It is well known that a finite group $G$ cannot be the union of two proper subgroups. In fact, an easy counting argument shows that such a union covers at most three quarters of the elements of $G$. It is equally well known that $G$ is never a union of conjugates of a proper subgroup $H$. Cameron-Cohen [4] have shown more precisely that there are at least $|H|$ elements outside such a union. On the other hand, it may happen that $G$ is covered by $n \geq 3$ arbitrary proper subgroups $H_{1}, \ldots, H_{n} \leq G$. While many authors classified such groups for a given $n$ (the interested reader is referred to the survey [2]), we are interested in the situation where $H_{1} \cup \ldots \cup H_{n} \neq G$. We show that a portion of elements, depending only on $n$, lies outside this union. In fact, this holds more generally for union of cosets of subgroups. To the authors' knowledge, this has apparently not been observed in the literature. In the first part of the paper,

Benjamin Sambale

sambale@math.uni-hannover.de

Marius Tărnăuceanu

tarnauc@uaic.ro

1 Institut für Algebra, Zahlentheorie und Diskrete Mathematik, Leibniz Universität Hannover, Welfengarten 1, 30167 Hannover, Germany

2 Faculty of Mathematics, “Al.I. Cuza” University, Iaşi, Romania 
we prove more precisely that $|G| /(2 n$ !) elements lie outside such a coset union. In the second part, we investigate our conjecture that even $|G| / 2^{n}$ elements lie outside the union. For elementary abelian groups, we obtain in Theorem 6 the best possible bound of that kind by using a linear algebra approach due to Alon-Füredi [1]. For cyclic groups, our results are closely related to a number theoretical problem by Erdős [5] (the details are outlined at the end of the paper). We like to mention that there are other open conjectures on union of cosets such as the long-standing Herzog-Schönheim Conjecture [6,8].

\section{Main result}

Theorem 1 For every positive integer $n$, there exists a constant $\gamma_{n}<1$ with the following property: For every finite group $G$ and every $n$ subgroups $H_{1}, \ldots, H_{n} \leq G$ and $g_{1}, \ldots, g_{n} \in G$ either $g_{1} H_{1} \cup \ldots \cup g_{n} H_{n}=G$ or $\left|g_{1} H_{1} \cup \ldots \cup g_{n} H_{n}\right| \leq \gamma_{n}|G|$.

Proof We argue by induction on $n$. For $n=1$, the claim holds with $\gamma_{1}=\frac{1}{2}$ by Lagrange's Theorem. Now let $n \geq 2, H_{1}, \ldots, H_{n} \leq G$ and $g_{1}, \ldots, g_{n} \in G$ such that $g_{1} H_{1} \cup \ldots \cup g_{n} H_{n} \neq G$. Let $s_{i}:=\left|G: H_{i}\right|$ for $i=1, \ldots, n$. We may assume that $s_{1} \leq \ldots \leq s_{n}$. Let $\alpha_{n}$ be the smallest positive integer such that $\gamma_{n-1}+\frac{1}{\alpha_{n}}<1$. If $s_{n} \geq \alpha_{n}$, then induction yields

$$
\begin{aligned}
\left|g_{1} H_{1} \cup \ldots \cup g_{n} H_{n}\right| & \leq\left|g_{1} H_{1} \cup \ldots \cup g_{n-1} H_{n-1}\right|+\left|g_{n} H_{n}\right| \\
& \leq\left(\gamma_{n-1}+\frac{1}{s_{n}}\right)|G| \leq\left(\gamma_{n-1}+\frac{1}{\alpha_{n}}\right)|G| .
\end{aligned}
$$

Now let $s_{n} \leq \alpha_{n}$ and $H:=H_{1} \cap \ldots \cap H_{n}$. Using Poincaré's formula $\left|G: H_{i} \cap H_{j}\right| \leq$ $\left|G: H_{i}\right|\left|G: H_{j}\right|$ repeatedly, we get $|G: H| \leq s_{1} \ldots s_{n} \leq \alpha_{n}^{n}$. Since $g_{1} H_{1} \cup \ldots \cup g_{n} H_{n}$ is a union of $H$-cosets, it follows that

$$
\left|g_{1} H_{1} \cup \ldots \cup g_{n} H_{n}\right| \leq|G|-|H| \leq\left(1-\frac{1}{\alpha_{n}^{n}}\right)|G| .
$$

Hence, the claim holds with

$$
\gamma_{n}:=\max \left\{\gamma_{n-1}+\frac{1}{\alpha_{n}}, 1-\frac{1}{\alpha_{n}^{n}}\right\}<1
$$

The proof of Theorem 1 yields only a very crude bound on $\gamma_{n}$. With some more effort, we can prove an effective bound as follows.

Proposition 2 Theorem 1 holds with $\gamma_{n}=\frac{2 n !-1}{2 n !}$.

Proof We reuse the notation from the proof of Theorem 1. We already know that the claim holds for $n=1$. Thus, let $n \geq 2$. If $n+1 \leq s_{1} \leq \ldots \leq s_{n}$, then

$$
\left|g_{1} H_{1} \cup \ldots \cup g_{n} H_{n}\right| \leq\left|H_{1}\right|+\cdots+\left|H_{n}\right| \leq \frac{n}{n+1}|G| \leq \frac{2 n !-1}{2 n !}|G|
$$


as desired.

Now let $s_{1} \leq n$. Since $G$ is the union of all cosets of $H_{1}$, there exists a coset $g H_{1}$ such that $g H_{1} \nsubseteq g_{1} H_{1} \cup \ldots \cup g_{n} H_{n}$. Since $\left|g_{1} H_{1} \cup \ldots \cup g_{n} H_{n}\right|=\left|g^{-1}\left(g_{1} H_{1} \cup \ldots \cup g_{n} H_{n}\right)\right|$, we may replace $g_{i}$ by $g_{i}^{\prime}:=g^{-1} g_{i}$ for $i=1, \ldots, n$. Then, $H_{1} \nsubseteq g_{1}^{\prime} H_{1} \cup \ldots \cup g_{n}^{\prime} H_{n}$ and $H_{1} \cap g_{1}^{\prime} H_{1}=\varnothing$. It follows that

$$
g_{1}^{\prime} H_{1} \cup \ldots \cup g_{n}^{\prime} H_{n} \subseteq\left(G \backslash H_{1}\right) \dot{\cup} \bigcup_{i=2}^{n}\left(g_{i}^{\prime} H_{i} \cap H_{1}\right)
$$

If $g_{i}^{\prime} H_{i} \cap H_{1} \neq \varnothing$, then $g_{i}^{\prime} H_{i} \cap H_{1}=h_{i}\left(H_{i} \cap H_{1}\right)$ for some $h_{i} \in H_{1}$. By induction on $n$, we conclude that

$$
\left|\left(G \backslash H_{1}\right) \dot{\cup} \bigcup_{i=2}^{n}\left(g_{i}^{\prime} H_{i} \cap H_{1}\right)\right| \leq \frac{s_{1}-1}{s_{1}}|G|+\gamma_{n-1}\left|H_{1}\right| \leq \frac{s_{1}+\gamma_{n-1}-1}{s_{1}}|G| .
$$

Since $\gamma_{n-1}-1<0$, it follows that

$$
\frac{s_{1}+\gamma_{n-1}-1}{s_{1}} \leq \frac{n+\gamma_{n-1}-1}{n}=\frac{2 n !+2(n-1) !-1-2(n-1) !}{2 n !}=\gamma_{n}
$$

as desired.

In most cases, cosets can cover more elements than subgroups. For instance, if $G$ is a $p$-group, then two distinct cosets of a maximal subgroup cover $\frac{2}{p}|G|$ elements while two distinct maximal subgroups only cover $\frac{2 p-1}{p^{2}}|G|$ elements (see also Theorem 6).

In order to compute a lower bound on $\gamma_{n}$, let us consider an elementary abelian 2-group $G=\left\langle x_{1}, \ldots, x_{n}\right\rangle$ rank $n$. Let $H_{i}:=\left\langle x_{j}: j \neq i\right\rangle$. Then, $H_{1} \cup \ldots \cup H_{n}=$ $G \backslash\left\{x_{1} \ldots x_{n}\right\}$, and therefore, $\gamma_{n} \geq\left(2^{n}-1\right) / 2^{n}$ for all $n \geq 1$. If we restrict ourselves to union of subgroups, we can show that this bound is indeed optimal for small $n$.

Proposition 3 For every finite group $G$ and every set of subgroups $H_{1}, \ldots, H_{n} \leq G$ with $n \leq 5$ either $H_{1} \cup \ldots \cup H_{n}=G$ or $\left|H_{1} \cup \ldots \cup H_{n}\right| \leq \frac{2^{n}-1}{2^{n}}|G|$. Equality can only hold if $\left|G: H_{1} \cap \ldots \cap H_{n}\right|=2^{n}$.

Proof We may assume that $n \geq 2, H_{1} \cup \ldots \cup H_{n} \neq G$ and $H_{i} \nsubseteq \bigcup_{j \neq i} H_{j}$ for $i=1, \ldots, n$. Let $N:=\{1, \ldots, n\}$ and $H_{I}:=\bigcap_{i \in I} H_{i}$ for $I \subseteq N$. Suppose first that $L:=H_{N \backslash\{i\}} \nsubseteq H_{i}$ for some $i$, say $i=1$. Let $U:=G \backslash \bigcup_{i=2}^{n} H_{i}$. By induction on $n$, we have $|U| \geq|G| / 2^{n-1}$. Moreover, $U$ is a union of $L$-cosets. If $g \in G$ and $x \in g L \cap H_{1}$, then

$$
\left|g L \cap H_{1}\right|=\left|x\left(L \cap H_{1}\right)\right|=\left|L \cap H_{1}\right|=\left|H_{N}\right| .
$$

Hence, $\left|U \cap H_{1}\right| \leq \sum_{g L \subseteq U}\left|H_{N}\right|=\frac{|U|}{|L|}\left|H_{N}\right|$. It follows that

$$
\left|G \backslash \bigcup_{i=1}^{n} H_{i}\right|=\left|U \backslash H_{1}\right|=|U|-\left|U \cap H_{1}\right| \geq|U|\left(1-\frac{1}{\left|L: H_{N}\right|}\right) \geq \frac{|G|}{2^{n}}
$$


as desired. Equality can only hold if $|U|=|G| / 2^{n-1}$ and $\left|L: H_{N}\right|=2$. In this case, induction yields $|G: L|=2^{n-1}$ and $\left|G: H_{N}\right|=2^{n}$.

Hence, in the following we will assume that $H_{N \backslash\{i\}} \subseteq H_{i}$ for $i=1, \ldots, n$. In particular, $n \geq 3$. Since $H_{1} \cup \ldots \cup H_{n}$ is a union of $H_{N}$-cosets, we may also assume that $\left|G: H_{N}\right|>2^{n}$ as in the proof of Theorem 1 . We need to show the strict inequality $\left|H_{1} \cup \ldots \cup H_{n}\right|<\frac{2^{n}-1}{2^{n}}|G|$. Using

$$
\left|H_{1} \cup \ldots \cup H_{n}\right| \leq\left|H_{1} \cup \ldots \cup H_{n-1}\right|+\left|H_{n}\right|-\left|H_{1} \cap H_{n}\right| \leq\left(\gamma_{n-1}+\frac{1}{s_{n}}-\frac{1}{s_{n}^{2}}\right)|G|
$$

and induction, the indices $s_{i}:=\left|G: H_{i}\right|$ can be bounded. In particular, there are only finitely many choices. By using

$$
\left|G: H_{I}\right||| G: H_{I \cup J}|\leq| H_{I \cap J}: H_{I}|| G: H_{J} \mid=\frac{\left|G: H_{I}\right|\left|G: H_{J}\right|}{\left|G: H_{I \cap J}\right|}
$$

for $I, J \subseteq N$, we can enumerate all possible indices $\left|G: H_{I}\right|$ for $I \subseteq N$ by computer. The claim can then be checked with the exclusion-inclusion principle. Note that for $n=3$ this becomes

$$
\left|H_{1} \cup H_{2} \cup H_{3}\right|=\left(\frac{1}{s_{1}}+\frac{1}{s_{2}}+\frac{1}{s_{3}}-\frac{2}{\left|G: H_{N}\right|}\right)|G|
$$

where $9 \leq\left|G: H_{N}\right|=\left|G: H_{1} \cap H_{2}\right| \leq s_{1} s_{2}$ and $s_{1} \leq s_{2} \leq s_{3}$. It is easy to see that this implies $\left|H_{1} \cup H_{2} \cup H_{3}\right| \leq \frac{7}{9}|G|$ with equality if and only if $s_{1}=s_{2}=s_{3}=3$. For $n=4$, we obtain similarly

$$
\left|H_{1} \cup \ldots \cup H_{4}\right| \leq\left(\frac{1}{s_{1}}+\cdots+\frac{1}{s_{4}}-\frac{1}{s_{1} s_{2}}-\frac{1}{s_{1} s_{3}}-\ldots-\frac{1}{s_{3} s_{4}}+\frac{3}{\left|G: H_{N}\right|}\right)|G|
$$

where $\left|G: H_{N}\right| \geq 17$ (in fact, $\left|G: H_{N}\right| \geq 18$ since $\left|G: H_{N}\right|$ cannot be a prime). By induction, we get $s_{4} \leq 15$. The maximum for the estimate above is assumed for $s_{1}=s_{2}=2, s_{3}=s_{4}=15$ (checked by computer). This maximum is again strictly less than $\frac{15}{16}|G|$.

Finally, let $n=5$. Here we first estimate the union of four out of five subgroups. This leaves us with a short list of exceptional cases. In all those cases, there exist subgroups $A, B, C, D \in\left\{H_{1}, \ldots, H_{5}\right\}$ with the following indices

$$
\begin{aligned}
|G: A \cap B| & =4, & |G: C \cap D| & =|G: C||G: D|, \\
|G: A \cap B \cap C| & =2|G: C|, & |G: A \cap B \cap D| & =2|G: D| .
\end{aligned}
$$

From $|G: A \cap B|=4$, we obtain $|G: A|=|G: B|=2$ and $A \cap B \unlhd G$ (the proof of Proposition 2 now already yields $\gamma_{5}=31 / 32$, but we need a strict inequality here). In particular, $(A \cap B) C \leq G$ with $|(A \cap B) C: A \cap B|=|C: A \cap B \cap C|=2$. Thus, $A, B,(A \cap B) C$ are the maximal subgroups of $G$ containing $A \cap B$, and therefore, 
$G=A \cup B \cup(A \cap B) C$. From $|G: A \cap B \cap D|=2|G: D|=|G: D \cap A|=|G: D \cap B|$ we obtain $D \cap A=D \cap B$. It follows that

$$
D=(D \cap A) \cup(D \cap B) \cup(D \cap(A \cap B) C)=(D \cap A) \cup(D \cap(A \cap B) C) .
$$

Since $D$ is not the union of two proper subgroups, we conclude that $D \subseteq(A \cap B) C$. But also $C \subseteq(A \cap B) C$. Now $G=C D \subseteq(A \cap B) C$, because $|G: C \cap D|=\mid G$ : $C \| G: D \mid$. Contradiction.

We remark that the following alternative procedure applies more generally to union of cosets. Note that $G$ acts on $\bigcup_{i=1}^{n} G / H_{i}$ by left multiplication. The kernel $N$ of this action is contained in $H_{1} \cap \ldots \cap H_{n}$. Since $g_{1} H_{1} \cup \ldots \cup g_{n} H_{n}$ is the union of the cosets in $g_{1}\left(H_{1} / N\right) \cup \ldots \cup g_{n}\left(H_{n} / N\right)$, we may replace $G$ by $G / N$. Then $G$ is isomorphic to a subgroup of a direct product of symmetric groups $\prod_{i=1}^{n} S_{\left|G: H_{i}\right|}$. In principle, we can enumerate those subgroups by computer, but doing so becomes impractical when $n$ is large.

\section{Nilpotent groups}

In order to extend Proposition 3 to other cases, we provide a reduction theorem for nilpotent groups. Let $\delta_{n}(G)$ be the largest constant such that $\left|G \backslash\left(g_{1} H_{1} \cup \ldots \cup g_{n} H_{n}\right)\right| \geq$ $\delta_{n}(G)|G|$ whenever $g_{1} H_{1} \cup \ldots \cup g_{n} H_{n} \neq G$. We wish to show that $\delta_{n}(G) \geq 1 / 2^{n}$.

Lemma 4 Let $n \geq 1$. Suppose that for every $p$-group $P$ and every $m \leq n$ we have $\delta_{m}(P) \geq 1 / 2^{m}$. Then, $\delta_{n}(G) \geq 1 / 2^{n}$ for every nilpotent group $G$.

Proof Let $G$ be a nilpotent group. Let $p_{1}, \ldots, p_{k}$ be the distinct prime divisors of $|G|$. Let $P_{i}:=\mathrm{O}_{p_{i}}(G)$ be the Sylow $p_{i}$-subgroup and $Q_{i}:=\mathrm{O}_{p_{i}^{\prime}}(G)$ its normal complement. Note that $G=P_{1} \times \cdots \times P_{k}$. Let $g_{1} H_{1}, \ldots, g_{n} H_{n}$ be cosets of subgroups of $G$ such that $g_{1} H_{1} \cup \ldots \cup g_{n} H_{n} \neq G$. Suppose that $\left|G: H_{1}\right|$ is divisible by $p_{i}$ and $p_{j}$ with $i \neq j$. Let $K:=H_{1} \mathrm{~N}_{P_{i}}\left(H_{1}\right)$ and $L:=H_{1} \mathrm{~N}_{P_{j}}\left(H_{1}\right)$. Then, $g_{1} H_{1}=g_{1}(K \cap L)=$ $g_{1} K \cap g_{1} L$ and $g_{1} K \cup g_{2} H_{2} \cup \ldots \cup g_{n} H_{n} \neq G$ or $g_{1} L \cup g_{2} H_{2} \cup \ldots \cup g_{n} H_{n} \neq G$. Thus, we may replace $H_{1}$ by $K$ or $L$, respectively. Since every subgroup of $G$ is subnormal, we may continue in this way until $\left|G: H_{1}\right|$ is a prime power. We repeat this process with $H_{i}$ for $i=2, \ldots, k$. Then, every $H_{i}$ contains a unique $Q_{j}$.

Let $\mathcal{H}_{i}:=\left\{H_{j}: Q_{i} \subseteq H_{j}\right\}$ for $i=1, \ldots, k$. Then, $\left\{H_{1}, \ldots, H_{n}\right\}$ is the disjoint union of $\mathcal{H}_{1}, \ldots, \mathcal{H}_{k}$. In particular, $n=\left|\mathcal{H}_{1}\right|+\cdots+\left|\mathcal{H}_{k}\right|$. Moreover, an element $\left(x_{1}, \ldots, x_{k}\right) \in P_{1} \times \cdots \times P_{k}$ does not lie in $g_{1} H_{1} \cup g_{2} H_{2} \cup \ldots \cup g_{n} H_{n}$ if and only if $x_{i} Q_{i}$ does not lie in $\bigcup_{H_{j} \in \mathcal{H}_{i}} g_{j}\left(H_{j} / Q_{i}\right)$ for $i=1, \ldots, k$. If we regard $H_{j} / Q_{i}$ as subgroups of $P_{i} \cong G / Q_{i}$, it follows that

$$
\left|G \backslash\left(g_{1} H_{1} \cup \ldots \cup g_{n} H_{n}\right)\right| \geq \prod_{i=1}^{k} \delta_{\left|\mathcal{H}_{i}\right|}\left(P_{i}\right)\left|P_{i}\right| \geq \prod_{i=1}^{k} \frac{\left|P_{i}\right|}{2^{\left|\mathcal{H}_{i}\right|}}=\frac{1}{2^{n}}|G| .
$$


Unfortunately, we are unable to prove $\delta_{n}(P) \geq 1 / 2^{n}$ for $p$-groups in general. Nevertheless, we provide an optimal bound for elementary abelian $p$-groups by making use of combinatorial theorems of Alon-Füredi [1] (see also Theorem [9]). The following variant of the Schwartz-Zippel Lemma is an explicit version of [1, Theorem 5].

Lemma 5 Let $p$ be a prime, and let $\alpha \in \mathbb{F}_{p}\left[X_{1}, \ldots, X_{k}\right]$ be a polynomial of total degree $d=a+b(p-1)$ where $0 \leq a \leq p-2$. If $\alpha$ does not vanish identically on $\mathbb{F}_{p}^{k}$, then $\alpha$ is non-zero on at least $\bar{p}^{k-b-1}(p-a)$ points of $\mathbb{F}_{p}^{k}$. This bound is best possible for $d \leq k(p-1)$.

Proof We argue by induction on $k$. Without loss of generality, we may assume that $k>b$. If $k=1$, then $\alpha$ has at most $d=a$ roots in $\mathbb{F}_{p}$, so it is nonzero on at least $p-a$ points. Now let $k \geq 2$. By Fermat's little theorem, $x^{p}=x$ for all $x \in \mathbb{F}_{p}$. Hence, we can reduce all powers of $X_{1}$ such that the degree of $\alpha$ in $X_{1}$ is at most $p-1$. This might decrease $d$, so the bound will be even stronger. For $x \in \mathbb{F}_{p}$, let

$$
\gamma_{x}:=\alpha\left(x, X_{2}, \ldots, X_{k}\right) \in \mathbb{F}_{p}\left[X_{2}, \ldots, X_{k}\right]
$$

Let $C \subseteq \mathbb{F}_{p}$ be the set of $x \in \mathbb{F}_{p}$ such that $\gamma_{x}$ does not vanish identically on $\mathbb{F}_{p}^{k-1}$. By hypothesis, $C \neq \varnothing$. Let $p^{\prime}:=p-|C|$. Let

$$
\alpha=\alpha_{1} X_{1}^{p-1}+\alpha_{2} X_{1}^{p-2}+\cdots+\alpha_{p}
$$

with $\alpha_{i} \in \mathbb{F}_{p}\left[X_{2}, \ldots, X_{k}\right]$ and $\operatorname{deg}\left(\alpha_{i}\right) \leq d-p+i$ for $i=1, \ldots, p$. We arrange the elements of $\mathbb{F}_{p}^{k-1}$ in some fixed order, say $\mathbb{F}_{p}^{k-1}=\left\{v_{1}, v_{2}, \ldots, v_{p^{k-1}}\right\}$, and define $\overline{\alpha_{i}}:=\left(\alpha_{i}\left(v_{1}\right), \alpha_{i}\left(v_{2}\right), \ldots, \alpha_{i}\left(v_{p^{k-1}}\right)\right)^{\mathrm{t}} \in \mathbb{F}^{p^{k-1} \times 1}$. For $x \in \mathbb{F}_{p} \backslash C$, we obtain a linear equation $x^{p-1} \overline{\alpha_{1}}+x^{p-2} \overline{\alpha_{2}}+\cdots+\overline{\alpha_{p}}=0$. The Vandermonde matrix $A:=\left(x^{i}: i=\right.$ $\left.0, \ldots, p^{\prime}-1, x \in \mathbb{F}_{p} \backslash C\right)$ is invertible and

$$
\left(\overline{\alpha_{p}}, \overline{\alpha_{p-1}}, \ldots, \overline{\alpha_{|C|+1}}\right) A=-\left(x^{p-1} \overline{\alpha_{1}}+\cdots+x^{p^{\prime}} \overline{\alpha_{|C|}}: x \in \mathbb{F}_{p} \backslash C\right)
$$

Therefore, we can express the vectors $\overline{\alpha_{|C|+1}}, \ldots, \overline{\alpha_{p}}$ as linear combinations of $\left\{x^{p-1 \overline{\alpha_{1}}}+\cdots+x^{p^{\prime}} \overline{\alpha_{|C|}}: x \in \mathbb{F}_{p} \backslash C\right\}$. Hence, we may replace each $\alpha_{i}$ with $|C|<i \leq p$ by a linear combination of $\alpha_{1}, \ldots, \alpha_{|C|}$ without changing the values on $\mathbb{F}_{p}^{k-1}$. Eventually, $\operatorname{deg}\left(\alpha_{i}\right) \leq d-p^{\prime}$ for all $i$ and $\operatorname{deg}\left(\gamma_{x}\right) \leq d-p^{\prime}$ for $x \in C$. By induction, $\gamma_{x}$ is non-zero on at least $p^{k-b^{\prime}-2}\left(p-a^{\prime}\right)$ points of $\mathbb{F}_{p}^{k-1}$ where $d-p^{\prime}=a^{\prime}+b^{\prime}(p-1)$ with $0 \leq a^{\prime} \leq p-2$. Consequently, $\alpha$ is nonzero on at least

$$
|C| p^{k-b^{\prime}-2}\left(p-a^{\prime}\right)=p^{k-b^{\prime}-2}\left(p-a^{\prime}\right)\left(p-p^{\prime}\right)
$$

points of $\mathbb{F}_{p}^{k}$.

Suppose first that $p^{\prime} \leq a$. Then, $a^{\prime}=a-p^{\prime}$ and $b^{\prime}=b$. It follows that

$$
p^{k-b-2}\left(p-a+p^{\prime}\right)\left(p-p^{\prime}\right) \geq p^{k-b-2}\left(p-a+p^{\prime}\right)(p-a) \geq p^{k-b-1}(p-a)
$$


and we are done. Now let $a<p^{\prime} \leq p-1$. Then, $a^{\prime}=a-p^{\prime}+p-1$ and $b^{\prime}=b-1$. Since $\left(p^{\prime}-a\right)\left(p-p^{\prime}\right) \geq p^{\prime}-a$, we obtain $\left(p^{\prime}-a+1\right)\left(p-p^{\prime}\right) \geq p-a$. This yields $p^{k-b^{\prime}-2}\left(p-a^{\prime}\right)\left(p-p^{\prime}\right) \geq p^{k-b-1}(p-a)$ as desired.

To see that the bound is best possible, just consider

$$
\alpha=\prod_{i=1}^{b}\left(X_{i}^{p-1}-1\right) \prod_{j=1}^{a}\left(X_{b+1}-j\right)
$$

where $j$ is interpreted as $1+\cdots+1 \in \mathbb{F}_{p}$ ( $j$ summands $)$.

Theorem 6 Let $G$ be an elementary abelian p-group, $H_{1}, \ldots, H_{n} \leq G$ and $g_{1}, \ldots, g_{n} \in G$ such that $g_{1} H_{1} \cup \ldots \cup g_{n} H_{n} \neq G$. Let $n=a+b(p-1)$ where $0 \leq a \leq p-2$. Then,

$$
\left|g_{1} H_{1} \cup \ldots \cup g_{n} H_{n}\right| \leq \frac{p^{b+1}-p+a}{p^{b+1}}|G| \leq \frac{2^{n}-1}{2^{n}}|G|
$$

and the first inequality is best possible.

Proof We regard $G$ as the $\mathbb{F}_{p}$-vector space $\mathbb{F}_{p}^{k}$. Each coset $g_{i} H_{i}$ is the set of solutions of a linear system $A_{i} x=b_{i}$. By hypothesis, there exists $x \in G \backslash\left(g_{1} H_{1} \cup \ldots \cup g_{n} H_{n}\right)$. For each $i$, we choose a row $a_{i}$ of $A_{i}$ such that $a_{i} x \neq \beta_{i}$ where $\beta_{i} \in \mathbb{F}_{p}$ is the corresponding entry of $b_{i}$. Then, the polynomial

$$
\alpha\left(X_{1}, \ldots, X_{k}\right):=\prod_{i=1}^{n}\left(a_{i}\left(X_{1}, \ldots, X_{k}\right)^{\mathrm{t}}-\beta_{i}\right) \in \mathbb{F}_{p}\left[X_{1}, \ldots, X_{k}\right]
$$

of degree $n$ does not vanish on $x$. By Lemma $5, \alpha$ is nonzero on at least $p^{k-b-1}(p-a)=$ $\frac{p-a}{p^{b+1}}|G|$ points of $G$. All these points lie outside of $g_{1} H_{1} \cup \ldots \cup g_{n} H_{n}$. This implies the first inequality. For the second, we may assume that $a=p-2$ and $b+1=$ $\frac{n-a}{p-1}+1=\frac{n+1}{p-1}$. It suffices to show that $2^{n+1} \geq p^{b+1}$, i. e. $(n+1) \log _{p}(2) \geq \frac{n+1}{p-1}$. This is true since $2^{p-1} \geq p$.

In order to show that the first inequality is optimal, we choose $H_{1}=\ldots=H_{p-1}$ as a maximal subgroup of $G$ and $g_{1}, \ldots, g_{p-1} \in G$ such that $G \backslash H_{1}=g_{1} H_{1} \cup \ldots \cup$ $g_{p-1} H_{1}$. Similarly, choose $H_{p}=\ldots=H_{2 p-1}$ as a maximal subgroup of $H_{1}$ and $g_{p}, \ldots, g_{2 p-1} \in H_{1}$ such that $H_{1} \backslash H_{p}=g_{p} H_{p} \cup \ldots \cup g_{2 p-1} H_{p}$ and so on. This will certainly yield the exact bound.

We remark that Theorem 6 extends to arbitrary finite $p$-groups as long as $n \leq 2 p-2$. To see this, consider $g_{1} H_{1} \cup \ldots \cup g_{n} H_{n} \neq G$ where $G$ is a finite $p$-group. If all $H_{1}, \ldots, H_{n}$ are maximal subgroups of $G$, then, by the remark at the end of Sect. 2, we can go over to the elementary abelian group $G / \Phi(G)$ where $\Phi(G)$ is the Frattini subgroup of $G$. In this case, the claim follows from Theorem 6. Otherwise, we may 
assume that $H_{n}$ is not maximal. Then, the claim follows by induction on $n$, because

$$
\begin{aligned}
\left|g_{1} H_{1} \cup \ldots \cup g_{n} H_{n}\right| & \leq\left|g_{1} H_{1} \cup \ldots \cup g_{n-1} H_{n-1}\right|+\left|H_{n}\right| \\
\leq & \left|g_{1} H_{1} \cup \ldots \cup g_{n-1} H_{n-1}\right|+\frac{1}{p^{2}}|G| .
\end{aligned}
$$

On a different note, we mention that the subgroup lattice of some (but not all) $p$ groups can be embedded into the subgroup lattice of an elementary abelian $p$-group (see [3]). For instance, Theorem 6 carries over to cyclic $p$-groups (here, two cosets are either disjoint or one lies in the other). By virtue of Lemma 4 , the bound $\delta_{n}(G) \geq 1 / 2^{n}$ now holds for all cyclic groups. The reviewer pointed out that this is connected to the following arithmetical theorem of Erdős [5]: Suppose that $a_{1}+m_{1} \mathbb{Z}, \ldots, a_{n}+m_{n} \mathbb{Z}$ are pairwise disjoint residue classes such that $0<m_{1}<\ldots<m_{n}$. We may consider these residue classes as cosets inside $\mathbb{Z} / M \mathbb{Z}$ where $M:=\operatorname{lcm}\left(m_{1}, \ldots, m_{n}\right)$. Then, the union of these (disjoint) cosets is $\frac{M}{m_{1}}+\cdots+\frac{M}{m_{n}}$. Erdôs showed that $\frac{M}{m_{1}}+\cdots+\frac{M}{m_{n}} \leq \frac{2^{n}-1}{2^{n}} M$ which is precisely our bound.

On the basis of these examples, the following general conjecture seems reasonable.

\section{Conjecture 7 The best possible bound in Theorem 1 is $\gamma_{n}=\left(2^{n}-1\right) / 2^{n}$ for all $n$.}

Acknowledgements We thank an anonymous reviewer for his/her valuable comments. The first author is supported by the German Research Foundation (SA 2864/1-2 and SA 2864/3-1).

Funding Open Access funding enabled and organized by Projekt DEAL.

Open Access This article is licensed under a Creative Commons Attribution 4.0 International License, which permits use, sharing, adaptation, distribution and reproduction in any medium or format, as long as you give appropriate credit to the original author(s) and the source, provide a link to the Creative Commons licence, and indicate if changes were made. The images or other third party material in this article are included in the article's Creative Commons licence, unless indicated otherwise in a credit line to the material. If material is not included in the article's Creative Commons licence and your intended use is not permitted by statutory regulation or exceeds the permitted use, you will need to obtain permission directly from the copyright holder. To view a copy of this licence, visit http://creativecommons.org/licenses/by/4.0/.

\section{References}

1. Alon, N., Füredi, Z.: Covering the cube by affine hyperplanes. European J. Combin. 14, 79-83 (1993)

2. Bhargava, M.: Groups as unions of proper subgroups. Amer. Math. Monthly 116, 413-422 (2009)

3. Butler, L.M., Hales, A.W.: Generalized flags in p-groups. Comput. Math. Appl. 39, 67-76 (2000)

4. Cameron, P.J., Cohen, A.M.: On the number of fixed point free elements in a permutation group. Discrete Math. 106(107), 135-138 (1992)

5. Erdős, P.: Remarks on number theory. IV. Extremal problems in number theory. I (Hungarian). Mat. Lapok 13, 228-255 (1962)

6. Herzog, M., Schönheim, J.: Research problem no. 9. Canad. Math. Bull. 17, 150 (1974)

7. Lettl, G., Sun, Z.W.: On covers of abelian groups by cosets. Acta Arith 131, 341-350 (2008)

8. Sun, Z.W.: On the Herzog-Schönheim conjecture for uniform covers of groups. J. Algebra 273, 153-175 (2004)

9. Szegedy, B.: Coverings of abelian groups and vector spaces. J. Combin. Theory Ser. A 114, 20-34 (2007) 
Publisher's Note Springer Nature remains neutral with regard to jurisdictional claims in published maps and institutional affiliations. 